\title{
GENERAL THEORY OF CURVES ON RULED SURFACES*
}

BY

\section{E. J. WILCZYNSKI $†$}

§1. Relation between the differential equations of the surface and of the curves situated upon it.

Let a ruled surface be given by means of the system of differential equations

$$
\begin{aligned}
& y^{\prime \prime}+p_{11} y^{\prime}+p_{12} z^{\prime}+q_{11} y+q_{12} z=0, \\
& z^{\prime \prime}+p_{21} y^{\prime}+p_{22} z^{\prime}+q_{21} y+q_{22} z=0,
\end{aligned}
$$

so that the curves $C_{y}$ and $C_{z}$ will be two curves upon it, the lines joining corresponding points of these two curves being generators of the surface. We shall eliminate once $z$ and once $y$, so as to obtain the linear differential equations of the fourth order which each of these functions must satisfy.

We have from (1), by differentiation,

where

$$
\begin{aligned}
& y^{(3)}=r_{11} y^{\prime}+r_{12} z^{\prime}+s_{11} y+s_{12} z, \\
& z^{(3)}=r_{21} y^{\prime}+r_{22} z^{\prime}+s_{21} y+s_{22} z,
\end{aligned}
$$

$$
\begin{array}{ll}
r_{11}=p_{11}^{2}+p_{12} p_{21}-p_{11}^{\prime}-q_{11}, & s_{11}=p_{11} q_{11}+p_{12} q_{21}-q_{11}^{\prime}, \\
r_{12}=p_{12}\left(p_{11}+p_{22}\right)-p_{12}^{\prime}-q_{12}, & s_{12}=p_{11} q_{12}+p_{12} q_{22}-q_{12}^{\prime}, \\
r_{21}=p_{21}\left(p_{11}+p_{22}\right)-p_{21}^{\prime}-q_{21}, & s_{21}=p_{21} q_{11}+p_{22} q_{21}-q_{21}^{\prime}, \\
r_{22}=p_{22}^{2}+p_{12} p_{21}-p_{22}^{\prime}-q_{22}, & s_{22}=p_{21} q_{12}+p_{22} q_{22}-q_{22}^{\prime} .
\end{array}
$$

We find, by another differentiation,

where

$$
\begin{aligned}
& y^{(4)}=l_{11} y^{\prime}+l_{12} z^{\prime}+m_{11} y+m_{12} z, \\
& z^{(4)}=l_{21} y^{\prime}+l_{22} z^{\prime}+m_{21} y+m_{22} z,
\end{aligned}
$$

* Presented to the Society A pril 30, 1904. Received for publication March 12, 1904.

t Of the Carnegie Institution of Washington. 


$$
\begin{array}{ll}
l_{11}=-p_{11} r_{11}-p_{21} r_{12}+r_{11}^{\prime}+s_{11}, & m_{11}=-r_{11} q_{11}-r_{12} q_{21}+s_{11}^{\prime}, \\
l_{12}=-p_{12} r_{11}-p_{22} r_{12}+r_{12}^{\prime}+s_{12}, & m_{12}=-r_{11} q_{12}-r_{12} q_{22}+s_{12}^{\prime}, \\
l_{21}=-p_{11} r_{21}-p_{21} r_{22}+r_{21}^{\prime}+s_{21}, & m_{21}=-r_{21} q_{11}-r_{22} q_{21}+s_{21}^{\prime}, \\
l_{22}=-p_{12} r_{21}-p_{22} r_{22}+r_{22}^{\prime}+s_{22}, & m_{22}=-r_{21} q_{12}-r_{22} q_{22}+s_{22}^{\prime} .
\end{array}
$$

If we put further

$$
\Delta_{1}=p_{12} s_{12}-q_{12} r_{12}, \quad \Delta_{2}=p_{21} s_{21}-q_{21} r_{21},
$$

we can find, from the above equations,

$$
\begin{aligned}
\Delta_{1} z & =p_{12} y^{(3)}+r_{12} y^{\prime \prime}+\left(p_{11} r_{12}-p_{12} r_{11}\right) y^{\prime}+\left(q_{11} r_{12}-p_{12} s_{11}\right) y, \\
-\Delta_{1} z^{\prime} & =q_{12} y^{(3)}+s_{12} y^{\prime \prime}+\left(p_{11} s_{12}-q_{12} r_{11}\right) y^{\prime}+\left(q_{11} s_{12}-q_{12} s_{11}\right) y,
\end{aligned}
$$

and similarly,

$$
\begin{aligned}
\Delta_{2} y & =p_{21} z^{(3)}+r_{21} z^{\prime \prime}+\left(p_{22} r_{21}-p_{21} r_{22}\right) z^{\prime}+\left(q_{22} r_{21}-p_{21} s_{22}\right) z, \\
-\Delta_{2} y^{\prime} & =q_{21} z^{(3)}+s_{21} z^{\prime \prime}+\left(p_{22} s_{21}-q_{21} r_{22}\right) z^{\prime}+\left(q_{22} s_{21}-q_{21} s_{22}\right) z .
\end{aligned}
$$

Finally, we obtain the required differential equations for $y$ and $z$, viz:

and

$$
\begin{aligned}
\Delta_{1} y^{(4)}=\left(p_{12}\right. & \left.m_{12}-q_{12} l_{12}\right) y^{(3)}+\left(r_{12} m_{12}-s_{12} l_{12}\right) y^{\prime \prime} \\
& +\left[\left(p_{11} r_{12}-p_{12} r_{11}\right) m_{12}-\left(p_{11} s_{12}-q_{12} r_{11}\right) l_{12}+\Delta_{1} l_{11}\right] y^{\prime} \\
& +\left[\left(q_{11} r_{12}-p_{12} s_{11}\right) m_{12}-\left(q_{11} s_{12}-q_{12} s_{11}\right) l_{12}+\Delta_{1} m_{11}\right] y,
\end{aligned}
$$

$$
\begin{aligned}
\Delta_{2} z^{(4)}=\left(p_{21}\right. & \left.m_{21}-q_{21} l_{21}\right) z^{(3)}+\left(r_{21} m_{21}-s_{21} l_{21}\right) z^{\prime \prime} \\
& +\left[\left(p_{22} r_{21}-p_{21} r_{22}\right) m_{21}-\left(p_{22} s_{21}-q_{21} r_{22}\right) l_{21}+\Delta_{2} l_{22}\right] z^{\prime} \\
& +\left[\left(q_{22} r_{21}-p_{21} s_{22}\right) m_{21}-\left(q_{22} s_{21}-q_{21} s_{22}\right) l_{21}+\Delta_{2} m_{22}\right] z .
\end{aligned}
$$

These equations are capable of a vast number of applications. Any question, in fact, in regard to the existence of curves of a specified character on a ruled surface must make use of them.

We notice that the conditions $\Delta_{1}=0$ or $\Delta_{2}=0$ will be necessary and sufficient to make $C_{y}$ or $C_{z}$ plane curves; the differential equations (of the third order) of these plane curves are found by putting $\Delta_{1}=0$ or $\Delta_{2}=0$ in (7) or (8) respectively. We will merely indicate a few other applications of these formulas. Let us write (9), more briefly,

$$
y^{(4)}+4 p_{1} y^{(3)}+6 p_{2} y^{\prime \prime}+4 p_{3} y^{\prime}+p_{4} y=0 .
$$

It is easy to write down the conditions that the integral curve of $\left(9^{\prime}\right)$ shall belong to a linear complex, or that it shall be a twisted cubic. In one case its invariant of weight 3 , and in the other both of its invariants, must vanish. But 
these conditions, which we now find expressed in terms of the coefficients of (1), become conditions for a particular kind of ruled surface, which contains such curves. One can impose other conditions, for example, that these curves shall be flecnode curves or asymptotic curves on the surface, and then proceed to study the particular class of surface characterized.

It is not our intention to follow up any of these special problems, interesting as they are. We shall, however, apply our equations for the purpose of answering some questions of a fundamental nature in the general theory of ruled surfaces.

§2. On ruled surfaces, one of the branches of whose flecnode curve is given.

The flecnode curve is so important in the general theory of ruled surfaces, that it seems essential to investigate to what extent it may be arbitrarily assigned.

If one of the sheets of the flecnode surface, $F^{\prime}$, of $S$ is given, there remain only two possibilities for $S$, namely, one or the other of the two sheets of the flecnode surface of $F^{\prime}$. But let us suppose that we merely know that a certain curve $C$ is one of the branches of the flecnode curve on $S$. Then there are two questions to answer. Can this curve be chosen arbitrarily? And how far does it determine the surface $S$ ?

Let the curve $C$ be given by means of its differential equation

$$
\frac{d^{4} \bar{y}}{d \bar{x}^{\bar{t}}}+4 \bar{p}_{1} \frac{d^{3} \bar{y}}{d \bar{x}^{3}}+6 \bar{p}_{2} \frac{d^{2} \bar{y}}{d \bar{x}^{2}}+4 \bar{p}_{3} \frac{d \bar{y}}{d \bar{x}}+\bar{p}_{4} \bar{y}=0,
$$

where $\bar{p}_{1}, \ldots, \bar{p}_{4}$ are given functions of $\bar{x}$. In the system of differential equations (1) defining our surface $S$, we must regard the coefficients $p_{i k}$ and $q_{i k}$ as unknown functions. We may, however, assume without exception that $u_{12}=0$, so that $C_{y}$ is one of the branches of the flecnode curve on $S$, that $p_{21}=0$, so that $C_{z}$ is an asymptotic curve on $S$, and that $p_{11}=p_{22}=0$. Under these assumptions we form the differential equation (9) of the curve $C_{y}$. Since $C_{y}$ is to be identical with $C$ it must be possible to convert equation (9) into (11) by a transformation of the form

$$
y=\phi(x) \bar{y}, \quad \bar{x}=f^{\prime}(x) .
$$

The functions $\phi$ and $f$ are not independent however. For, while the equations $u_{12}=0$ and $p_{21}=0$ are not disturbed by any transformation of this form, the conditions $p_{11}=p_{22}=0$ are. In fact a transformation of the form (12) converts $(1)$ into another system of the same form whose corresponding coefficients $\bar{p}_{11}$ and $\bar{p}_{22}$ will be

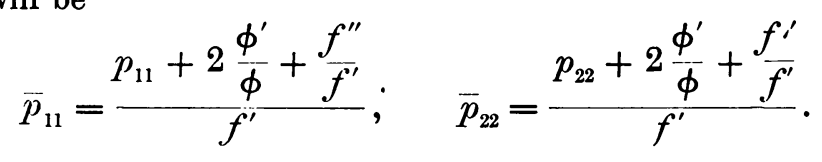


In order, therefore, that after this transformation $\bar{p}_{11}$ and $\bar{p}_{22}$ may again vanish, we must have

$$
\phi=\frac{C}{\sqrt{f^{\prime \prime}}},
$$

where $C$ is an arbitrary constant, which may be put equal to unity.

If then we apply the transformation (12) to (11), we shall get an equation

$$
\frac{d^{4} y}{d x^{4}}+4 p_{1} \frac{d^{3} y}{d x^{3}}+6 p_{2} \frac{d^{2} y}{d x^{2}}+4 p_{3} \frac{d y}{d x}+p_{4} y=0,
$$

which we must identify with (9). Equating coefficients gives us a system of four equations with five unknown functions of $x$, viz.: $f^{\prime}, p_{12}, q_{11}, q_{21}, q_{22}$.

We find, therefore, the following theorem: An arbitrary space curve being given, it can be considered as one branch of the flecnode curve of an infinity of ruled surfaces, into whose general expression there enters an arbitrary function. One may, therefore, impose another condition and still obtain an infinity of ruled surfaces.

The most general curve $C_{z}$ which is capable of being the second branch of the flecnode curve on a ruled surface for which $C_{y}$ is the first branch, involves, therefore, in its expression one arbitrary function. It cannot, therefore, be an arbitrary curve, as that would involve three arbitrary functions.

Therefore, two curves taken at random cannot be connected point to point in such a way as to constitute the complete flecnode curve upon the ruled surface thus generated.

We can also prove our theorem by purely synthetic considerations. Let us take points $P_{1}, P_{2}, P_{3}, P_{4}, \ldots$ on an arbitrary curve, corresponding for example to equal increments $\Delta x$ of the parameter. Through $P_{1}, P_{2}, P_{3}$ draw three arbitrary lines $g_{1}, g_{2}, g_{3}$. We can draw a line $f_{1}$ through $P_{1}$ intersecting $g_{2}$ and $g_{3}$, say in $Q_{2}$ and $Q_{3}$. Take an arbitrary point $Q_{4}$ on $f_{1}$, and join it to $P_{4}$ by a line $g_{4}$. Then $f_{1}$ intersects $g_{1}, g_{2}, g_{3}, g_{4}$. Through $P_{2}$ we draw a line $f_{2}$ intersecting $g_{3}$ and $g_{4}$ in points $Q_{3}^{\prime}, Q_{4}^{\prime}$ and of course $g_{2}$ in $Q_{2}^{\prime}=P_{2}$. Take an arbitrary point $Q_{5}^{\prime}$ on $f_{2}$ and join it to $P_{5}$ by a line $g_{5}$. Continue this process. Clearly, we shall get two assemblages of lines $g_{1}, g_{2}, \ldots$ and $f_{1}, f_{2}, \ldots$, which when $P_{1}, P_{2}, \ldots$ are taken closer and closer together, approach as a limit two ruled surfaces having the given curve as flecnode curve, and which are flecnode surfaces of each other. The first three lines $g_{1}, g_{2}, g_{3}$ are arbitrary, and thus give rise to six constants of integration. Further, the double ratios $\left(P_{1}, Q_{2}, Q_{3}, Q_{4}\right),\left(Q_{2}^{\prime}, Q_{3}^{\prime}, Q_{4}^{\prime}, Q_{5}^{\prime}\right)$, etc., may be chosen arbitrarily, which brings into evidence the arbitrary function involved in the construction of these surfaces.

The construction which has just been described becomes indeterminate if the given curve $C$ is a straight line. For then $Q_{4}$ coincides with $P_{4}$, etc. In fact, 
the most general ruled surface with a given straight line directrix depends on two arbitrary functions.

If the given curve $C$ is to be at the same time the second branch of the flecnode curve, i. e., if both of the branches of the flecnode curve of $S$ coincide with $C, g_{4}$ must be tangent to the hyperboloid determined by $g_{1}, g_{2}, g_{3} ; g_{5}$ must be tangent to the hyperboloid determined by $g_{2}, g_{3}, g_{4}$; etc. This condition, therefore, clearly fixes the double ratios $\left(Q_{1} Q_{2} Q_{3} Q_{4}\right)$, etc., i. e., the arbitrary function. Therefore this problem has in general $\infty^{6}$ solutions.

Let us assume that $C_{y}$ is not a straight line. Let us call the developable surface formed by the tangents of $C_{y}$ its primary developable. There exists another important developable surface containing $C_{y}$, which we shall speak of as its secondary developable, as indicated in the following theorem.

1. If at every point of the flecnode curve of $S$ there be drawn the generator of the surface, the flecnode tangent, the tangent of the flecnode curve, and finally the line which is the harmonic conjugate of the latter with respect to the other two, the locus of these last lines is a developable surface, the secondary developable of the flecnode curve.

2. We can find a single infinity of ruled surfaces, each having one branch of its flecnode curve in common with that of $S$. This family of $\infty^{1}$ surfaces can be described as an involution, of which any surface of the family and its flecnode surface form a pair. The primary and secondary developables of the branch of the flecnode surface considered, are the double surfaces of this involution. In fact, the generators of these surfaces, at every point of their common flecnode curve, form an involution in the usual sense.

We proceed to prove these theorems. Since $C_{y}$ is a branch of the flecnode curve, we may assume $u_{12}=p_{11}=p_{22}=0$. System (1) assumes the form

$$
y^{\prime \prime}+p_{12} z^{\prime}+q_{11} y+\frac{1}{2} p_{12}^{\prime} z=0, \quad z^{\prime \prime}+p_{21} y^{\prime}+q_{21} y+q_{22} z=0 .
$$

The flecnode tangent at $P_{y}$ is the line joining $P_{y}$ to $P_{\rho}$, where

$$
\rho=2 y^{\prime}+p_{12} z,
$$

while the tangent of the flecnode curve joins $P_{y}$ to $P_{y^{\prime}}$. In the plane pencil formed by these lines, the harmonic conjugate of $P_{y} P_{y^{\prime}}$ with respect to $P_{y} P_{z}$ and $P_{y} P_{\rho}$ will be the line $P_{y} P_{\tau}$, where

$$
\tau=y^{\prime}+p_{12} z \text {. }
$$

But from the first equation of (14) we find

$$
\tau^{\prime}+q_{11} y-\frac{1}{2} \frac{p_{12}^{\prime}}{p_{12}}\left(\tau-y^{\prime}\right)=0
$$

i. e., $P_{y} P_{\tau}$ generates a developable surface as asserted in the first theorem. 
Put

$$
\begin{aligned}
& e=\tau+k y^{\prime}=(1+k) y^{\prime}+p_{12} z, \\
& f=\tau-k y^{\prime}=(1-k) y^{\prime}+p_{12} z,
\end{aligned}
$$

where $k$ is a constant. Clearly the lines $P_{y} P_{e}$ and $P_{y} P_{f}$ form a pair of the involution whose double lines are $P_{y} P_{y^{\prime}}$ and $P_{y} P_{\tau}$.

One finds that $y$ and $e$ satisfy the following system of differential equations :

$$
\begin{aligned}
& y^{\prime \prime}+P_{11} y^{\prime}+P_{12} e^{\prime}+Q_{11} y+Q_{12} e=0, \\
& e^{\prime \prime}+P_{21} y^{\prime}+P_{22} e^{\prime}+Q_{21} y+Q_{22} e=0,
\end{aligned}
$$

where

$$
\begin{aligned}
P_{11} & =-\frac{1+k}{2 k} \frac{p_{12}^{\prime}}{p_{12}}, \quad P_{12}=-\frac{1}{k}, \quad Q_{11}=-\frac{q_{11}}{k}, \quad Q_{12}=\frac{1}{2 k} \frac{p_{12}^{\prime}}{p_{12}}, \\
P_{21} & =(1+k) q_{11}+k(1+k) q_{22}-k p_{12} p_{21}+\frac{(1-3 k)\left(1-k^{2}\right)}{4 k}\left(\frac{p_{12}^{\prime}}{p_{12}}\right)^{2}+\frac{1-k^{2} p_{12}^{\prime \prime}}{2} \frac{p_{12}}{p^{\prime}}, \\
(18) P_{22} & =\frac{1-3 k}{2 k} \frac{p_{12}^{\prime}}{p_{12}}, \\
Q_{21} & \left.=(1+k) q_{11}^{\prime}-k p_{12} q_{21}+\frac{(1-3 k)(1+k) \frac{p_{12}^{\prime}}{p_{12}} q_{11},}{2 k} \frac{(1-3 k)(1-k)}{4 k}\left(\frac{p_{12}^{\prime}}{p_{12}}\right)^{2}+\frac{1-k}{2} \frac{p_{12}^{\prime \prime}}{p_{12}}\right] .
\end{aligned}
$$

We find

$$
U_{12}=2 P_{12}^{\prime}-4 Q_{12}+P_{12}\left(P_{11}+P_{22}\right)=0,
$$

i. e., the curve $C_{y}$ is flecnode curve on the ruled surface $S_{k}$ generated by $P_{y} P_{e}$. The flecnode surface of $S_{k}$ is obtained by joining $P_{y}$ to the point

$$
2 y^{\prime}+P_{11} y+P_{12} e=-\frac{1}{k} f-\frac{1+k}{2 k} \frac{p_{12}^{\prime}}{p_{12}} y,
$$

a point on the line $P_{y} P_{f}$. We see therefore that the ruled surfaces $S_{k}$ and and $S_{-k}$ are flecnode surfaces of each other. We have now proved our second theorem, and we may speak of an involution of ruled surfaces having one branch of their flecnode curve in common. The double surfaces of the involution are developables, while the members of each pair of the involution are flecnode surfaces of each other.

We have seen that $P_{y} P_{\tau}$.generates a developable. If

$$
g=\alpha y+\beta \tau
$$

represents its edge of regression, it must be possible to represent $g^{\prime}$ in the form

$$
g^{\prime}=\gamma y+\delta \tau,
$$

since the line $P_{y} P_{\tau}$ must then be tangent to the curve $C_{g}$. 
We find, by differentiation, making use of (15),

$$
g^{\prime}=\alpha y^{\prime}+\beta\left(\frac{1}{2} \frac{p_{12}^{\prime}}{p_{12}} \tau-q_{11} y-\frac{1}{2} \frac{p_{12}^{\prime}}{p_{12}} y^{\prime}\right)+\alpha^{\prime} y+\beta^{\prime} \tau,
$$

so that $g^{\prime}$ will be of the required form if, and only if,

Therefore

$$
\alpha: \beta=p_{12}^{\prime}: 2 p_{12} \text {. }
$$

$$
g=p_{12}^{\prime} y+2 p_{12} \tau \text {. }
$$

If we express $\tau$ in terms of $y, z$ and $\rho$, we shall find

$$
g=p_{12} \rho+p_{12}^{\prime} y+p_{12}^{2} z
$$

as the expression for the edge of regression of the secondury develinable of the branch $C_{y}$ of the flecnode curve. Similarly, if $\theta_{4} \neq 0$,

$$
h=p_{21} \sigma+p_{21}^{\prime} z+p_{21}^{2} y
$$

will represent the cuspidal edge of the secondary developable of the branch $C_{*}$ of the flecnode curve, assuming, of course, $u_{21}=0$.

One easily finds

$$
-{ }_{2}^{3} p_{12}^{\prime} g+p_{12} g^{\prime}=\lambda y, \quad-{ }_{2}^{3} p_{21}^{\prime} h+p_{21} h^{\prime}=\mu z,
$$

where

$$
\begin{aligned}
& \lambda=p_{12} p_{12}^{\prime \prime}+\frac{1}{2} p_{12}^{2} u_{11}-p_{12}^{3} p_{21}-\frac{3}{2}\left(p_{12}^{\prime}\right)^{2}, \\
& \mu=p_{21} p_{21}^{\prime \prime}+\frac{1}{2} p_{21}^{2} u_{22}-p_{21}^{3} p_{12}-\frac{3}{2}\left(p_{21}^{\prime}\right)^{2} .
\end{aligned}
$$

The system of differential equations of which $g$ and $h$ are the solutions has the coefficients

$$
\begin{aligned}
& P_{11}=-\frac{1}{\lambda}\left[\lambda^{\prime}+\frac{3}{4} p_{12}^{2} p_{12}^{\prime} p_{21}\right], \quad P_{12}=\frac{p_{21} \lambda}{\mu}, \quad Q_{12}=-\frac{3 p_{21}^{\prime} \lambda}{2 \mu_{j}}, \\
& Q_{11}=-\frac{1}{\lambda p_{12}}\left[\lambda\left(2 p_{12}^{\prime \prime}+\frac{1}{4} p_{12} u_{11}-\frac{1}{2} p_{12}^{2} p_{21}\right)-\frac{3}{2} p_{12}^{\prime}\left(\lambda^{\prime}+\frac{3}{4} p_{12}^{2} p_{12}^{\prime} p_{21}\right)\right],
\end{aligned}
$$

while $P_{21}, P_{22}, Q_{22}, Q_{21}$ are obtained from these same equations by permuting the indices 1 and 2 , and consequently also the letters $\lambda$ and $\mu$.

We see that we thus obtain, corresponding uniquely to any ruled surface whose flecnode curve intersects every generator in two distinct points, another ruled surface which is generated by the lines joining corresponding points of the edges of regression of the secondary developables of the two branches of the flecnode curve.

Equations (20) show that one of the secondary developables of $C_{y}$ and $C_{z}$ degenerates into a cone if $\lambda$ or $\mu$ vanishes. In that case our new ruled surface also becomes a cone. If both of the secondary developables are cones, this ruled 
surface degenerates into the straight line joining their vertices. If $\lambda=\Delta_{1}=0$, the secondary developable of $C_{y}$ is a plane pencil.

Equations (22) show that this new ruled surface cannot be developable except if $\lambda$ or $\mu$ is zero, i. e., unless it is a cone. For the possibility $p_{12}=0$ or $p_{21}=0$ is to be excluded, since we should then have a ruled surface $S$ with a straight line directrix.

\section{§3. On ruled surfaces one of the branches of whose complex curve is given.}

There exists an infinity of ruled surfaces each of which contains an arbitrarily given curve as one branch of its complex curve. Into the general analytical expression of these surfaces there enters an arbitrary function. The analytical proof of this statement is precisely similar to that of the corresponding theorem of $\S 2$. We shall give at once a geometrical construction for these surfaces.

Let us consider five straight lines $g_{1}, \ldots, g_{5}$. Let $f_{1}^{\prime}, f_{1}^{\prime \prime}$ be the two transversals of $g_{1}, \cdots, g_{4}$, and $f_{2}^{\prime}, f_{2}^{\prime \prime}$ those of $g_{2}, \ldots, g_{5}$. Clearly $g_{1}, \ldots, g_{5}$ determine a linear complex, with respect to which $f_{1}^{\prime}, f_{1}^{\prime \prime}$ and $f_{2}^{\prime}, f_{2}^{\prime \prime}$ are two pairs of reciprocal polars. Take a point $P$ on $g_{1}$. The plane, which corresponds to it in the linear complex, passes through $g_{1}$ and the line $h$, which passes through $P$ and intersects both $f_{2}^{\prime}$ and $f_{2}^{\prime \prime}$. If $g_{2}, \ldots, g_{5}$ are made to approach each other, we shall have in the limit five consecutive generators of a ruled surface and its osculating linear complex. The plane tangent to this ruled surface at $P$ is the limit of the plane containing $g_{1}$ and the line through $P$ which intersect $g_{2}$ and $g_{3}$, i. e., the asymptotic tangent of the surface at $P$. If $P$ is a point on the complex curve, $h_{1}$ must be in the plane tangent to the ruled surface at $P$.

Now let an arbitrary curve be given, and let us choose points upon it, $P_{1}, P_{2}, P_{3}, \cdots$, according to any law. Through $P_{1}, \ldots, P_{4}$ draw four arbitrary lines $g_{1}, \cdots, g_{4}$. Through $P_{1}$ draw $h_{1}$, any line which intersects $g_{2}$. Let $Q$ be this point of intersection. The line $g_{5}$, through $P_{5}$, is to be constructed in such a way that the two transversals of $g_{2}, \ldots, g_{5}$ shall both meet $h_{1}$. Now these transversals must be generators of the second set on the hyperboloid determined by $g_{2}, g_{3}, g_{4}$. They must, therefore, be those two generators of the second set, $f_{2}^{\prime}$ and $f_{2}^{\prime \prime}$, which pass through the two points in which $h_{1}$ intersects the hyperboloid. One of these points is $Q$. There exists just one line through $P_{5}$ intersecting both $f_{2}^{\prime}$ and $f_{2}^{\prime \prime}$. It is the line $g_{5}$. In the same way, starting with $g_{2}, \cdots, g_{5}$, we can construct $g_{6}$, etc. Finally we pass to the limit. There enters an arbitrary function, fixing the position of the successive lines $h_{1}, h_{2}, \ldots$ in the planes in which they must lie.

That a corresponding theorem is true for asymptotic curves, is obvious.

NICE, Feliruary 25th, 1904. 